\title{
Electrothermoconvective instability in a heat generating dielectric fluid layer ${ }^{\text {is }}$
}

\author{
I.S. Shivakumara ${ }^{\mathrm{a}, *}$, M.S. Nagashree ${ }^{\mathrm{b}}$, K. Hemalatha ${ }^{\mathrm{c}}$ \\ ${ }^{\text {a }}$ UGC-Centre for Advanced Studies in Fluid Mechanics, Department of Mathematics, Bangalore University, Bangalore 560 001, India \\ ${ }^{\mathrm{b}}$ Department of Mathematics, Vijaya College, Bangalore-560 004, India \\ ${ }^{\mathrm{c}}$ Department of Mathematics, Mount Carmel College, Bangalore-560 052, India
}

Available online 11 June 2007

\begin{abstract}
The combined effects of vertical d.c. electric field and uniform internal heat generation on the onset of convection in a horizontal poorly conducting dielectric fluid layer heated uniformly from below are investigated. The Galerkin method is used to solve the resulting eigenvalue problem. Parametric study is conducted to know the effects of varying electric Rayleigh number, $R_{e}$ and dimensionless heat source strength, $N_{s}$. It is found that these two parameters exhibit a dual effect on the onset of natural convection; a result noticed in contrast to their effects when they are acting in isolation.
\end{abstract}

(C) 2007 Elsevier Ltd. All rights reserved.

Keywords: Dielectric fluid; d.c. electric field; Thermoelectroconvection; Heat generation

\section{Introduction}

The problem of Rayleigh-Benard instability, which is due to gravitational force caused by vertical density stratification, and also the effect of a uniform vertical magnetic field on such thermal instability, called magnetoconvection, has been studied extensively by Chandrasekhar [1]. Besides, several studies have also been carried out to assess the effect of electric field on natural convection due to the fact that many problems of practical importance involve poorly conducting dielectric fluids wherein electric forces essentially govern the motion rather than the magnetic forces. Roberts [2] was the first to make an individual study on electrohydrodynamic convection by considering the dielectric constant as well as electrical conductivity as a linear function of temperature. Turnbull [3], Takashima and Aldridge [4], Martin and Richardson [5], Maekawa et al. [6], Pontiga and Castellanos [7], Douiebe et al. [8] and Othman [9] have studied natural convection in the presence of an applied electric field.

Nonetheless, in electrothermoconvective instability problems, it is pertinent that in a dielectric fluid the applied electric field increases the vibrations of the atoms and eventually this will cause Joule heating throughout the fluid due to the flow of current. It is but natural to question how this heating affects the onset of convection in dielectric fluids under an applied

\footnotetext{
t5 Communicated by A.R. Balakrishnan and S. Jayanti.

* Corresponding author.

E-mail address: isshivakumara@hotmail.com (I.S. Shivakumara).
} 
electric field. This can aptly be modeled as volumetric heat generation within the dielectric fluid causing basic temperature distribution to be nonlinear which in turn has a profound effect on the stability of the system. The onset of thermal instability in a horizontal ordinary viscous fluid layer, subject to an internal heat generation, has been analyzed by Sparrow et al. [10] and Shivakumara and Suma [11]. In the latter paper an additional effect of vertical throughflow is also considered.

However, it is of interest and importance to know in what way the stability of the dielectric fluid layer would be affected in the simultaneous presence of electric field and volumetric heat generation which have not been given due attention in the literature. This has motivated the present study. The present work is of interest in the design of efficient heat exchangers and also in image processing devices which involve electric fields and fluids [5]. In these problems, the flow is likely to become unstable due to variation in temperature and hence examining its instability gains importance. From a geophysical standpoint, it is important that the electric field and internal heat generation together can provide the driving force in the laboratory models of thermal convection of electrically conducting fluids in the Earth's core [4]. Moreover, the present study also throws light to understand atmospheric electricity and control of thermal convective instability by means of conductivity variation induced by non-uniform thermal gradients [8]. In the present study, the linear stability theory is applied and the resulting eigenvalue problem is solved numerically.

\section{Mathematical formulation}

We consider a horizontal poorly conducting dielectric fluid layer of characteristic thickness $d$ with a uniformly distributed volumetric internal heat generation within the fluid. A Cartesian coordinate system with the $z$-axis pointing vertically upward is chosen such that the origin is at the bottom of the layer and the boundaries are assumed to be rigid. The lower boundary $z=0$ is maintained at a constant temperature $T_{0}+\Delta T / 2$ and a constant electric potential $\varphi_{0}-\Delta \varphi / 2$, while the upper boundary $z=d$ is maintained at a constant temperature $T_{0}-\Delta T / 2$ and a constant electric potential $\varphi_{0}+\Delta \varphi / 2$ with $\Delta T>0$ and $\Delta \varphi>0$. The governing equations are [2]:

$$
\begin{aligned}
& \nabla \cdot \vec{q}=0 \\
& \rho_{0}\left[\frac{\partial \vec{q}}{\partial t}+(\vec{q} \cdot \nabla) \vec{q}\right]=-\nabla p+\rho \vec{g}+\mu \nabla^{2} \vec{q}+\overrightarrow{f_{e}} \\
& \frac{\partial T}{\partial t}+(\vec{q} \cdot \nabla) T=\kappa \nabla^{2} T+Q \\
& \rho=\rho_{0}\left\{1-\alpha\left(T-T_{0}\right)\right\},
\end{aligned}
$$

where $\vec{q}(=u, v, w)$ is the velocity vector, $T$ the temperature, $p$ the pressure, $\rho$ the fluid density, $\mu$ the fluid viscosity, $\alpha$ the thermal expansion coefficient, $\rho_{0}$ the fluid density at $T=T_{0}, \kappa$ the thermal diffusivity, $Q$ the uniformly distributed volumetric internal heat generation in the fluid layer and $\vec{f}_{e}$ is the force of electrical origin which can be expressed as

$$
\vec{f}_{e}=\rho_{e} \vec{E}-\frac{1}{2} \vec{E} \cdot \vec{E} \nabla \varepsilon+\frac{1}{2} \nabla\left(\rho \frac{\partial \varepsilon}{\partial \rho} \vec{E} \cdot \vec{E}\right) .
$$

Here, $\rho_{e}$ is the charge density, $\varepsilon$ is the dielectric constant and $\vec{E}$ is the electric field. In Eq. (5), the first term on the right hand side is the Coulomb force which involves the free charge and the last two terms represent forces induced by the nonuniformity of the dielectric constant. We note that the last term can be absorbed into pressure and it has no effect on an incompressible fluid.

Since the fluid is poorly electrically conducting, the magnetic field effect is negligible and the Maxwell equations become

$$
\begin{aligned}
& \nabla \times \vec{E}=0 \text { or } \vec{E}=-\nabla \varphi \\
& \frac{\partial \rho_{e}}{\partial_{t}}+\nabla \cdot \vec{J}=0, \vec{J}=\sigma \vec{E}+\rho_{e} \vec{q}, \rho_{e}=\nabla \cdot(\varepsilon \vec{E})
\end{aligned}
$$


where $\varphi$ is the electric potential, $\vec{J}$ is the current density and $\sigma$ is the electrical conductivity. Since the problem is investigated under d.c. electric field, the Coulomb force becomes more dominant than the dielectric force [6]. Under the circumstances, the dielectric force is neglected (i.e., $\varepsilon=$ constant) and the electrical conductivity is assumed to vary linearly with temperature in the form $\sigma=\sigma_{0}\left[1+\beta\left(T-T_{0}\right)\right]$, where $\beta(>0)$ is the thermal coefficient of electrical conductivity. The basic steady state is assumed to be quiescent and consider the solution of the form $\vec{q}=0, p=p_{\mathrm{b}}(z), T=T_{\mathrm{b}}(z), \varphi=\varphi_{\mathrm{b}}(z)$, where the subscript $\mathrm{b}$ denotes the basic state. The unperturbed basic temperature and the electric potential are found to be

$$
T_{\mathrm{b}}(z)=T_{0}+\Delta T\left(\frac{1}{2}-\frac{z}{d}\right)+\frac{Q d^{2}}{2 \kappa}\left(\frac{z}{d}-\frac{z^{2}}{d^{2}}\right), \varphi_{\mathrm{b}}(z)=\varphi_{0}-\Delta \varphi\left(\frac{1}{2}-\frac{z}{d}\right)
$$

where we have assumed $\beta \ll 1$. It may be noted that the basic temperature distribution is nonlinear with the fluid layer height due to internal heat generation, and which has important implications on the stability of the system.

To study the stability of the basic state, we perturb the variables in the form

$$
\vec{q}=\vec{q}^{\prime}, p=p_{\mathrm{b}}(z)+p^{\prime}, T=T_{\mathrm{b}}(z)+T^{\prime}, \varphi=\varphi_{\mathrm{b}}(z)+\varphi^{\prime}
$$

Substituting Eq. (9) into the governing equations, linearzing, eliminating the pressure from the momentum equation by operating curl twice and retaining the vertical component, we obtain the following perturbed equations (after ignoring the primes):

$$
\begin{aligned}
& \rho_{0} \frac{\partial}{\partial_{t}}\left(\nabla^{2} w\right)=\alpha \rho_{0} g \nabla_{1}^{2} T+\frac{\varepsilon \Delta \varphi}{d} \nabla^{2} \nabla_{1}^{2} \varphi+\mu \nabla^{4} w \\
& \frac{\partial T}{\partial t}-\kappa \nabla^{2} T=-\frac{\mathrm{d} T_{\mathrm{b}}}{\mathrm{d} z} w \\
& \varepsilon \frac{\partial}{\partial t}\left(\nabla^{2} \varphi\right)=-\sigma_{0} \nabla^{2} \varphi-\sigma_{0} \frac{\beta \Delta \varphi}{d} \frac{\partial T}{\partial z} .
\end{aligned}
$$

On introducing the following dimensionless quantities $\left(x^{*}, y^{*}, z^{*}\right)=(x / d, y / d, z / d), w^{*}=w /(\kappa / d), t^{*}=t /\left(d^{2} / \kappa\right)$, $T^{*}=T / \Delta T$ and $\varphi^{*}=\varphi /(\beta \Delta \phi \Delta T)$ in Eqs. (10)-(12) and neglecting the asterisks for simplicity, we obtain

$$
\begin{aligned}
& \frac{1}{\operatorname{Pr}} \frac{\partial}{\partial t}\left(\nabla^{2} w\right)=R_{t} \nabla_{1}^{2} T+R_{e} \nabla^{2} \nabla_{1}^{2} \varphi+\nabla^{4} w \\
& \frac{\partial T}{\partial t}-\nabla^{2} T=-f(z) w \\
& \operatorname{Pe} \frac{\partial}{\partial t}\left(\nabla^{2} \varphi\right)+\nabla^{2} \varphi=-\frac{\partial T}{\partial z}
\end{aligned}
$$

In the above equations $R_{t}=\alpha g \Delta T d^{3} / v \kappa$ and $R_{e}=\varepsilon(\Delta \phi)^{2} \beta \Delta T / \mu \kappa$ are respectively, the thermal and electric Rayleigh numbers, $P r=v / \kappa$ is the Prandtl number, $P e=\varepsilon \kappa / \sigma_{0} d^{2}$ is the normalized thermal diffusivity, and

$$
f(z)=\mathrm{d} T_{\mathrm{b}} / \mathrm{d} z=N_{s}(1-2 z)-1
$$

is the basic temperature gradient, where $N_{s}=Q d^{2} / 2 \kappa \Delta T$ is the dimensionless heat source strength. The boundaries are assumed to be rigid with fixed temperature and electric potential and hence the associated boundary conditions are

$$
w=\partial w / \partial z=0=\theta=\varphi \text { at } z=0,1
$$


We now assume the normal mode solution for the dependent variables in the form

$$
(w, T, \varphi)=[W, \Theta, \Phi] e^{i(\ell x+m y)+\omega t}
$$

where $\omega$ is the growth rate, $\ell$ and $m$ are the wave numbers in the $x$ and $y$-directions, respectively. Substituting Eq. (18) in Eqs. (13)-(15) and noting that the principle of exchange of stability is valid [2,6] (i.e., $\omega=0)$, we arrive at the following stability equations (after eliminating $\Phi$ from the momentum equation):

$$
\begin{aligned}
& \left(D^{2}-a^{2}\right)^{2} W=R_{t} a^{2} \Theta+R_{e} a^{2} D \Theta \\
& \left(D^{2}-a^{2}\right) \Theta=f(z) W .
\end{aligned}
$$

where $D=\mathrm{d} / \mathrm{d} z$ and $a=\sqrt{l^{2}+m^{2}}$ is the overall horizontal wave number.

The required boundary conditions are

$$
W=D W=\Theta=0 \text { at } z=0,1
$$

\section{Method of solution}

Eqs. (19) and (20) with boundary conditions (21) constitute an eigenvalue problem with $R_{t}$ or $R_{e}$ as an eigenvalue. The resulting eigenvalue problem is solved numerically using Galerkin method. Accordingly, the unknown variables are written in a series of base functions as

$$
W=\sum_{i=1}^{N} A_{i} W_{i}, \quad \Theta=\sum_{i=1}^{N} B_{i} \Theta_{i}
$$

where $A_{i}$ and $B_{i}$ are constants and the base functions $W_{i}$ and $\Theta_{i}$ are represented by modified Tchebychev polynomials as trial functions satisfying the boundary conditions. Substituting Eq. (22) into Eqs. (19) and (20) and the Galerkin procedure of demanding that the residues be orthogonal to the basis functions applied, we get the following system of homogeneous algebraic equations.

$$
\begin{aligned}
& C_{j i} A_{i}+D_{j i} B_{i}=0 \\
& E_{j i} A_{i}+F_{j i} B_{i}=0 .
\end{aligned}
$$

The coefficients $C_{j i}$ to $F_{j i}$ involve inner products of the basis functions and are given by

$$
\begin{aligned}
& C_{j i}=<D^{2} W_{j} D^{2} W_{i}+2 a^{2} D W_{j} D W_{i}+a^{4} W_{j} W i>; D_{j i}=-R_{t} a^{2}<W_{j} \Theta_{i}>-R_{e} a^{2}<D W_{j} \Theta_{i}> \\
& E_{j i}=<f(z) \Theta_{j} W_{i}>; F_{j i}=<D \Theta_{j} D \Theta_{i}+a^{2} \Theta_{j} \Theta_{i}>
\end{aligned}
$$

where the inner product is defined as $\langle f g\rangle=\int_{0}^{1} f g \mathrm{~d} z$.

\section{Numerical calculations and discussion}

The determinental equation, formed from Eqs. (23a) and (23b) for the existence of non-trivial solution, is solved numerically to obtain the critical Rayleigh number $R_{t c}$ as a function of the wave number $a$ for different values of $R_{e}$ and $N_{s}$. The inner products involved in the elements of the determinant are evaluated analytically rather than numerically to avoid errors in the numerical integration. It is noted that eight terms (i.e., $N=8$ ) in the series expansion of Eq. (22) are sufficient to achieve the convergence within an error of $0.001 \%$ for all the choices of parametric values. To validate the solution procedure, computations are carried out first under the limiting conditions of $R_{e}=0$ and also when $N_{s}=0$. The critical Rayleigh number $R_{t c}$ obtained for $R_{e}=0$ and for various values of $N_{s}$ are compared with the results of Sparrow 
Table 1

Comparison of $R_{t c}$ and $a_{c}$ for different values of $N_{s}$ with $R_{e}=0$

\begin{tabular}{|c|c|c|c|c|}
\hline \multicolumn{3}{|c|}{ Sparrow et al. [10] } & \multicolumn{2}{|c|}{ Present study } \\
\hline$N_{s}$ & $R_{t c}$ & $a_{c}$ & $R_{t c}$ & $a_{c}$ \\
\hline 0 & 1707.765 & 3.12 & 1707.762 & 3.113 \\
\hline 0.5 & 1704.453 & 3.121 & 1704.53 & 3.119 \\
\hline 1.0 & 1694.953 & 3.13 & 1694.95 & 3.127 \\
\hline 5.0 & 1462.863 & 3.30 & 1462.86 & 3.304 \\
\hline 10.0 & 1118.480 & 3.53 & 1118.43 & 3.529 \\
\hline 15.0 & 878.339 & 3.68 & 878.303 & 3.659 \\
\hline 20.0 & 717.201 & 3.74 & 717.20 & 3.736 \\
\hline 30.0 & 521.403 & 3.82 & 521.403 & 3.819 \\
\hline 40.0 & 408.558 & 3.86 & 408.558 & 3.863 \\
\hline 70.0 & 247.075 & 3.92 & 247.075 & 3.920 \\
\hline 100.0 & 176.936 & 3.94 & 176.936 & 3.944 \\
\hline
\end{tabular}

et al. [10] in Table 1, while $R_{t c}$ obtained for $N_{s}=0$ and for different values of $R_{e}$ are compared with those of Roberts [2] in Table 2. From the tables we note that the agreement is excellent and thus verifies the accuracy of the method employed.

We now look into simultaneous presence of electric force $\left(R_{e}\right)$ and internal heating $\left(N_{s}\right)$ which have opposing effect on the stability of the system. It is observed that these two mechanisms compensate each other in some cases or dominate over one another depending on their strength to behave in an unusual manner and the same is evident from Figs. 1 and 2). Fig. 1(a) shows that the critical Rayleigh number, $R_{t c}$ decreases monotonically with $N_{s}$ when $R_{e}=0$ (see also Table 1) and thus its primary effect is to promote instability because of the increase in energy supply to the system. To the contrary, $R_{t c}$ is increasing, rather than decreasing, with $N_{s}$ initially when the electric field is present (i.e., for $R_{e} \neq 0$ ) but soon it starts decreasing as $N_{s}$ values are further increased. In other words, the internal heat generation initially shows some stabilizing effect on thermally unstable dielectric fluid layer when the electric field is present; a result not noticed in the absence of electric field. As is evident from Fig. 1a, there are crossings of curves among different values of $R_{e}$. In particular, the crossing of $R_{e}=2000$ curve over the other curves may be attributed to the fact that increase in $R_{e}$ leads to increase in electric field strength which in turn generates large Joule heating within the dielectric fluid layer and hence causes more instability due to available additional heat energy to the system. One can also notice a similar trend for $R_{e}=1000$. In this case, the crossing of curve is shifted towards higher value of $N_{s}$ due to decrease in electric field strength. Although the critical wave number increases with an increase in the value of $N_{s}$ when $R_{e}=0$, a mixed type of variation in $a_{c}$ could be seen when $R_{e} \neq 0$ (see Fig. 1b). We note that, $a_{c}$ decreases initially with an increase in the value of $N_{s}$; again starts increasing and reaches a maximum value, then decreases with further increase in the value of $N_{s}$.

Fig. 2a is a plot of $R_{t c}$ versus $R_{e}$ for different values of $N_{s}$. From the figure we note that $R_{t c}$ increases with $R_{e}$ when $N_{s}=0$ (see also Table 2) and thus the primary effect of d.c. electric field is to delay the onset of convection. In contrast to this, $R_{t c}$ decreases when once $R_{e}$ exceeds a certain value; which in turn depends on the strength of internal heat generation (i.e., for $N_{s} \neq 0$ ). Thus the electric field has a destabilizing effect on the system in the presence of internal

Table 2

Comparison of $R_{t c}$ and $a_{c}$ for different values of $R_{e}$ with $N_{s}=0$

\begin{tabular}{lllll}
\hline Roberts [2] & & & Present study \\
\cline { 1 - 2 }$R_{e}$ & $R_{t c}$ & $a_{c}$ & $R_{t c}$ & 1707.76 \\
0 & 1707.762 & 3.116 & 1722.83 & 3.113 \\
200 & 1722.826 & 3.112 & 1767.66 & 3.104 \\
400 & 1767.670 & 3.068 & 1841.09 & 3.068 \\
600 & 1841.106 & 3.008 & 1941.14 & 2.926 \\
800 & 1941.160 & 2.926 & 2065.00 & 2.824 \\
1000 & 2065.034 & 2.824 & $a_{c}$ & \\
\hline
\end{tabular}


(a)

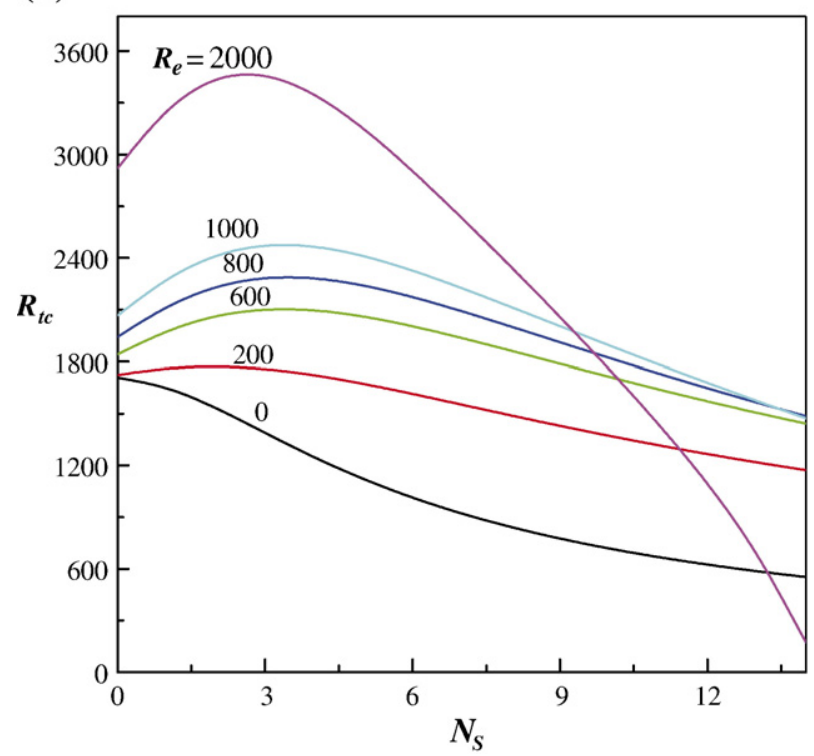

(b)

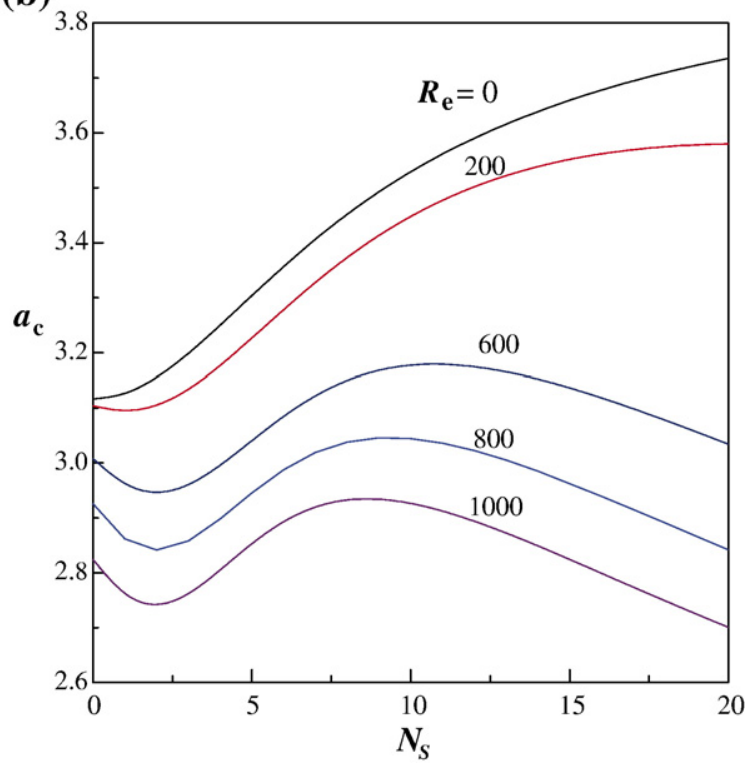

Fig. 1. (a) Variation of critical thermal Rayleigh number with $N_{s}$ for different values of $R_{e}$. (b).Variation of critical wave number with $N_{s}$ for different values of $R_{e}$.

heat generation and this could be attributed to the nonlinearity of the basic temperature distribution due to internal heat generation. The reason for the crossing of curve can be explained based on the same argument put forth previously. The effect of increasing $R_{e}$ is to decrease the critical wave number and hence its effect is to increase the size of convection cells (Fig. 2b).

(a)

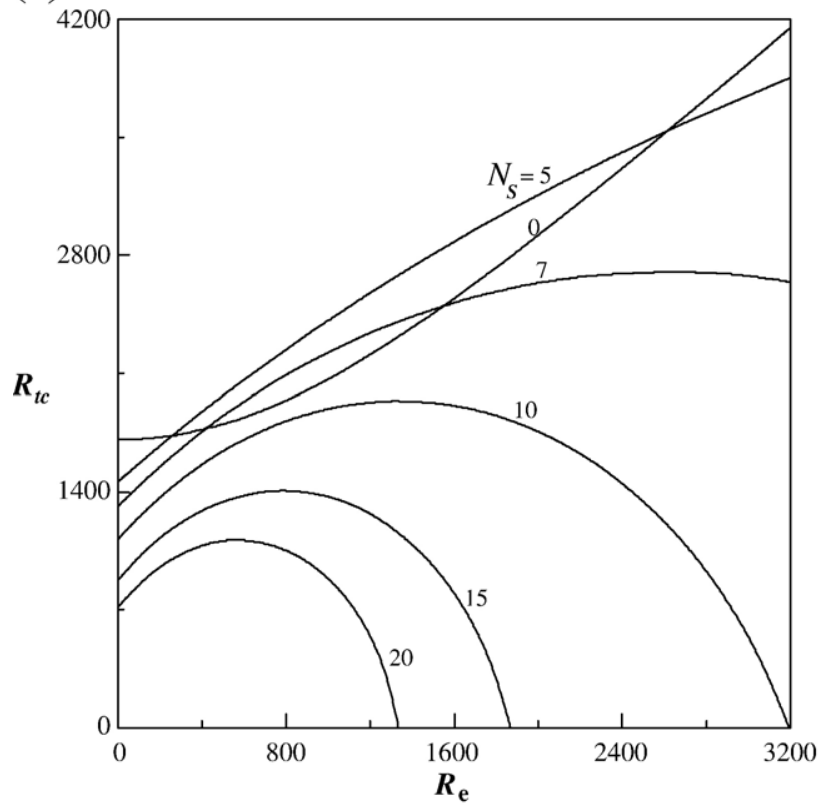

(b)

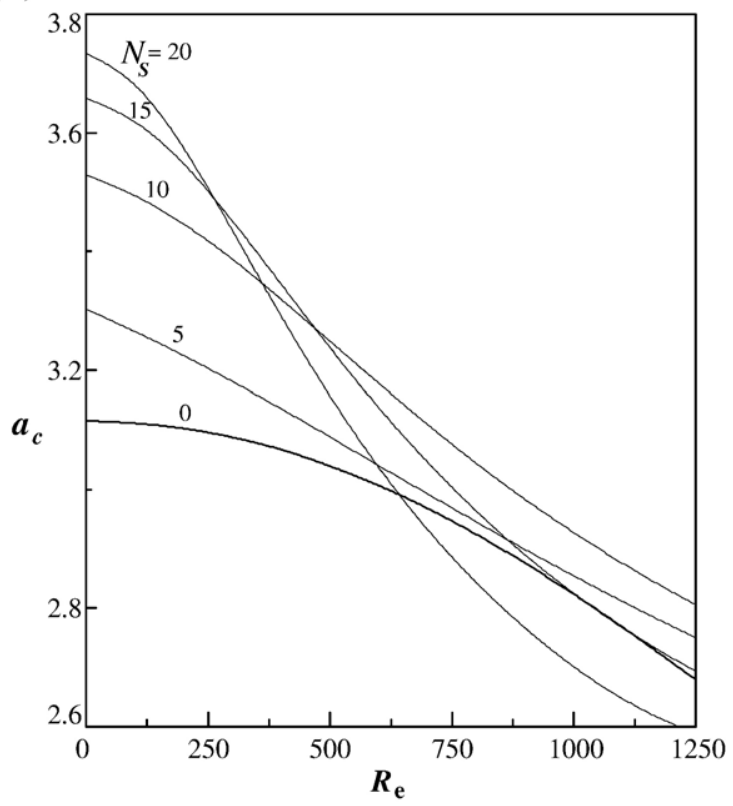

Fig. 2. (a).Variation of critical thermal Rayleigh number with $R_{e}$ for different values of $N_{s}$. (b).Variation of critical wave number with $R_{e}$ for different values of $N_{s}$. 


\section{Conclusion}

In the present paper we have analyzed the simultaneous effects of d.c. electric field and uniform internal heat generation on the onset of convection in a poorly conducting dielectric fluid layer heated from below. Contrary to their individual behavior, it is observed interestingly that an increase in the value of $R_{e}$ and also $N_{s}$ can have destabilizing and stabilizing effects, respectively on the system when they are acting together. The critical wave number decreases with an increase in the electric Rayleigh number for a fixed value of $N_{s}$, while it passes through a minimum and a maximum with an increase in the heat source strength for a fixed value of $R_{e}$.

\section{Acknowledgement}

This work was supported by the UGC-Centre for Advanced Studies in Fluid Mechanics.

\section{References}

[1] S. Chandrasekhar, Hydrodynamic and Hydromagnetic Stability, Clerendon Press, 1961.

[2] P.H. Roberts, Electrohydrodynamic convection, Quarterly Journal of Mechanics and Applied Mathematics 22 (1969) 211-220.

[3] R.J. Turnbull, Electroconvective instability with a stabilizing temperature gradient, I and II: theory and experimental results, Physics of Fluids 11 (1968) 2588-2603.

[4] M. Takashima, K.D. Aldridge, The stability of a horizontal layer of dielectric fluid under the simultaneous action of a vertical d.c. electric field and vertical temperature gradient, Quarterly Journal of Mechanics and Applied Mathematics 29 (1976) 71-87.

[5] P.J. Martin, A.T. Richardson, Conductivity models of electrothermal convection in a plane layer of dielectric liquid, Journal of Heat Transfer 106 (1984) 131-136.

[6] T. Maekawa, K. Abe, I. Tanasawa, Onset of natural convection under an electric field, International Journal of Heat and Mass Transfer 35 (1992) 613-621.

[7] F. Pontiga, A. Castellanos, Physical mechanisms of instability in a liquid layer subjected to an electric field and a thermal gradient, Physics of Fluids 6 (1994) 1684-1701.

[8] A. Douiebe, M. Hannaoui, G. Lebon, A. Benaboud, A. Khmou, Effects of a.c. electric field and rotation on Benard-Marangoni convection, Flow, Turbulence and Combustion 67 (2001) 185-204.

[9] M.I. Othman, Electrohydrodynamic instability of a rotating layer of a viscoelastic fluid heated from below, ZAMP 55 (2004) $468-482$.

[10] E.M. Sparrow, R.J. Goldstein, V.K. Jonsson, Thermal instability in a horizontal fluid layer: effect of boundary conditions and non-linear temperature profile, Journal of Fluid Mechanics 18 (1964) 513-529.

[11] I.S. Shivakumara, S.P. Suma, Effects of throughflow and internal heat generation on the onset of convection in a fluid layer, Acta Mechanica 140 (2000) 207-217. 